\title{
Utilization of Sustainable Resources for Promoting Energy Efficiency in Environment Using Smart Technologies
}

\author{
V. Vedanarayanan, ${ }^{1}$ Chirag Vibhakar, ${ }^{2}$ A. Sujaatha, ${ }^{3}$ Jiten K. Chavda, ${ }^{4}$ M. Karthik ${ }^{1 D},{ }^{5}$ \\ P. V. Pramila, ${ }^{6}$ and Ishwarya Komalnu Raghavan ${ }^{7}{ }^{7}$ \\ ${ }^{1}$ Department of Electronics and Communication Engineering, Sathyabama Institute of Science and Technology, Chennai, \\ 600119 Tamil Nadu, India \\ ${ }^{2}$ Department of Electrical Engineering, Gujarat Power Engineering \& Research Institute, Mehsana, 382710 Gujarat, India \\ ${ }^{3}$ Department of Civil Engineering, Sri Sairam Engineering College, Chennai, 600044 Tamil Nadu, India \\ ${ }^{4}$ Department of Electrical and Electronics Engineering, LD College of Engineering, Navrangpura, Ahmedabad 380015, India \\ ${ }^{5}$ Department of Electronics and Electronics Engineering, Kongu Engineering College, Perundurai, Erode, 638060 Tamil Nadu, India \\ ${ }^{6}$ Department of Computer Science and Engineering SIMTAS, Saveetha University, Chennai, Tamil Nadu, India \\ ${ }^{7}$ Department of Electromechanical Engineering, Faculty of Manufacturing, Institute of Technology, Hawassa University, Ethiopia
}

Correspondence should be addressed to Ishwarya Komalnu Raghavan; ishwarya138@hu.edu.et

Received 10 November 2021; Accepted 27 December 2021; Published 21 February 2022

Academic Editor: V. Mohanavel

Copyright (C) 2022 V. Vedanarayanan et al. This is an open access article distributed under the Creative Commons Attribution License, which permits unrestricted use, distribution, and reproduction in any medium, provided the original work is properly cited.

\begin{abstract}
Smart technology has potential in tracking the key challenges based on population based on the sustainable future. In today's cultures, a smart approach enables for the integration of information needed to address crucial concerns. The critical challenge is to limit the effects of global warming while maintaining a balanced economic growth. The impact of global warming mitigates the fundamental problem while ensuring the balance economic development. Intense research efforts should be directed toward balanced resource utilization, renewable energy system integration, efficient energy conversion technologies, effective process integration, effective techniques to enable a circular economy framework, and other issues that are important to the population. This paper finds the latest technology in the field of smart grid technologies which focused on the effective enhancement and efficient utilization of resource. The issues and challenges in using sustainable future are discussed and bring new ideas towards the sustainable future base on the application of smart technologies.
\end{abstract}

\section{Introduction}

Global energy demand is the worldwide concern, which is rapidly growing and satisfies the energy demand in the future. Economic growth, modernization, and automation are mainly depending on the energy supply. The carbon dioxide emission causes greenhouse effect and environmental degradation which threatened the global environment. Worldwide greenhouse gas emissions are steadily increasing on a global scale, where climate change concerns are becoming increasingly prevalent and wreaking havoc on the people. $\mathrm{CO}_{2}$ emissions have grown by around 2.7 percent, making them the most dominant emissions. This recent surge in $\mathrm{CO}_{2}$ emissions is very alarming, especially given that $\mathrm{CO}_{2}$ levels were nearly steady. $\mathrm{CO}_{2}$ levels are currently at $411 \mathrm{ppm}$, although they were below $400 \mathrm{ppm}$ just five years ago. Methane $\left(\mathrm{CH}_{4}\right)$ is the more dangerous greenhouse gas, as it is over 30 times more effective as a heat-trapping gas than $\mathrm{CO}_{2}$. Large amounts of $\mathrm{CH}_{4}$ are stored in ice sheets, where they are progressively released into the atmosphere when the ice sheets melt owing to climate change concerns. It is obvious that current measures are insufficient and that strong and timely global actions are required to attain this aim and, as a result, to limit the adverse effects of anthropogenic activities on the environment. It is the requirement for long-term development that leads to the integration of cost- 
effective ways based on interdisciplinary knowledge. Globally, fossil fuels are still used to generate power to a great level, accounting for more than half of all electricity generation, mostly coal, and natural gas. One of the major problems with current fossil fuel energy systems is their low conversion efficiency, which results in a high rate of thermal energy waste [1].

The renewable energy generation level increases as the energy development, which relies on the reduction of fossil fuel generation. The fossil fuel is taken as a primary source of energy to satisfy the supply of energy demand. This fossil fuel emits greenhouse gases and create hazard to environment and affect the future generation. So, the sustainable energy resource is of great significance to reduce the fossil fuel consumption. The renewable energy resources are free from emission. The renewable energy with smart technology provides an idea for the emission problem and contributes significantly in generating power with less emission of greenhouse gases. Increased energy demand, along with the depletion of fossil fuels, has heightened the need for rapid development of renewable energy (RE) sources to meet demand. However, the use of renewable energy as a percentage of total energy consumption in many nations is still at a low level [2]. RE is needed for a variety of activities, including lighting, heating, and cooking, and it may originate from a variety of sources, including biomass, solar, wind, and hydropower. The costs of developing various alternative energy sources to meet the energy needs of a certain location are varied [3].

The costs of developing various alternative energy sources to meet the energy needs of a certain location are varied. The challenge in energy resource management is deciding how to effectively employ available renewable energy resources to satisfy power demand [4]. Energy efficiency is widely acknowledged as a critical tactic in energy and climate change policy. Energy efficiency advantages include decreased greenhouse gas emissions and local air pollution, as well as fewer energy infrastructure expenditures, lower fossil fuel reliance, better competitiveness, and improved customer welfare [5].

For coal-based power plants, an average energy conversion efficiency of 35 percent to 45 percent is appropriate, with the latter number for state-of-the-art facilities, but for natural gas combined cycle plants, the efficiency can reach 60 percent. As a result, the operation of present fossil fuelbased technologies is accompanied by a high rate of emitted waste heat, ranging from $60 \%$ to $70 \%$ on average, which is mostly unutilized. In order to make better use of discharged waste heat, more efficient energy ideas are required, particularly in industrial applications.

Renewable energy technologies now available provide a viable alternative to fossil fuels, but with limits, owing to poor energy conversion efficiency and power generating nondispatchability. Photovoltaics (PV) and wind generators have dominated the market in the previous decade, with substantial growth, while the capacities of large hydroelectric plants have grown at a slower pace, since their potential has been fully used for decades, especially in developed nations.
Meanwhile, emission-free energy sources such as solar, wind, hydro, geothermal, biomass, and others exist across the world. Renewable energy technologies are an excellent alternative since they can considerably contribute to global power generation while emitting less greenhouse emissions. According to the International Energy Agency's (IEA) "sustainable future" scenario, renewable energy sources would generate 57 percent of global power by 2050 . To attain this ultimate goal, long-term forecasting and planning are essential. For a given zone, renewable energy-based power generation and delivery to the national grid are required. The traditional grid connects several networks, and the regulatory system is made up of different layers of communication and coordination, with the majority of the systems being regulated manually. A smart grid is a novel idea that enables the transmission and distribution grids to be improved. The smart grid system is the digitalization of transmission and the creation of new markets for renewable energy generation. In today's energy generating and distribution business, smart grid is frequently used [6].

A smart grid is a novel idea based on smart technology that allows the transmission and distribution system to be modernized. The smart grid technology is the digitalization of transmission and the creation of new markets for renewable energy generation. In today's energy generating and distribution business, the phrase "smart grid" is frequently used. The smart grid, when combined with distributed power generation, creates a new platform that dramatically improves electric energy security and quality [7]. As more energy sources become accessible, such as wind, hydropower, solar, and biomass, this approach becomes more feasible and trustworthy. Renewable and nonconventional energy sources are allowed to connect to a smart gridconnected distributed power generating connection. This paper explores the role of renewable energy sources in power generation and their integration with the smart grid system.

\section{Related Work}

Renewable energy sources must be integrated into the power system to achieve a variety of socioeconomic and environmental benefits, as well as to minimise greenhouse gas emissions from traditional power plants. PV power generation, on the other hand, is unpredictable and intermittent, putting significant pressure on the system and causing energy supply instability. Intermittent energy sources, to be more specific, may fail to assure the power supply's continuity and stability. Apart from the aforementioned concerns with PV integration, power system back-feeding poses substantial operational hurdles [8].

PV power producing equipment can cause reverse power flow, high voltage levels, and an increase in the number of tap operations. To cope with reverse power flow, dispersed PV power generation units have been proposed instead of centralised generating units. Furthermore, the integration of renewable power generation units as new distributed generations, encompassing large scale at the transmission level, medium scale at the distribution level, and small scale on 
commercial or residential buildings, can pose challenges for resource dispatch and control, as well as system operation [9].

The traditional approach to evaluating electric utility energy efficiency and smart-grid programmes, as well as an agent-based end-use modelling strategy that addresses many of the shortcomings of previous techniques, are also reviewed. Over a fifteen-year period, data from a representative sample of utility customers is utilized to evaluate energy efficiency and smart grid programme objectives. According to model analysis, combining the two least stringent efficiency and smart grid programme scenarios reduces peak hour demand by one-third more than the most stringent smart grid programme. This suggests that peak demand reductions are more feasible when both efficiency and smart grid programmes are considered [10].

Smart technology and energy approaches are being industrialized to enhance energy efficiency and decrease greenhouse gas emissions. Additionally, suggestions for incorporating energy-efficient and zero-carbon approach for lighting, air cooling, and conditioning, as well as sustainable energy consumption in office buildings around the country, are offered. The present policies and strategic energy of renewable energy technologies (RETs) are highlighted. The pronged method of lowering energy use, implementing RETs, and monitoring greenhouse gas emissions is used to decrease greenhouse gas emissions and enhance energy efficiency. Policy enactment, in addition to energy advocacy, has the potential to reduce greenhouse gas emissions, energy consumption, and efficiency of energy, all of which are important for humanity's future to be clean and sustainable [11].

Energy extraction, conversion, and usage processes are all prone to waste. Environmental deterioration, quicker depletion of energy supplies, and higher costs of energy products and services are all consequences of such waste. As a result, the notion of sustainable development requires a concerted effort to enhance energy efficiency in the production, conversion, and usage of energy. Energy resources and their use are inextricably linked to long-term development. Increasing the energy efficiency of operations that use sustainable energy resources is critical to achieving sustainable development [12].

The environment is another source of worry in the continuous use of fossil fuels, in addition to their limited nature and lack of viable replacement. Climate change and the environmental externalities linked with energy usage have emerged as a major global concern. The contribution of the industrial sector to greenhouse gas (GHG) emissions has been shown to be large, suggesting that reducing GHG emissions from the industry is one of the greatest approaches to address the climate change challenge. In this aspect, energy efficiency is critical. By enhancing the efficiency of energy usage in the sector, an estimated 10-30\% decrease can be accomplished at little or no expense [13].

Low-carbon technology, environmental protection, renewable energy, and green development have been at the forefront of global power system reform. New electricity market and power sector changes are being investigated, and renewable energy market mechanisms are being explored across the world to address climate change and emission reduction. Because the usage of hydropower production resources in industrialized nations has already reached a high level, renewable energy sources such as biomass, geothermal, win, and solar are being explored [14].

The majority of study on energy utilization has been concentrated on the residential sector. Energy behaviours in the residential sector are large field experiments assessing instruments to encourage more effective energy behaviours and seeking to discover behavioural drivers for energy use. In the last decade, psychology research has dominated the bulk of energy behaviour studies. It comprises a detailed investigation of the factors that influence proenvironmental behaviour in the residential sector, as well as the sorts of involvements that may be utilized to encourage it. Local and national governments utilize energy organisations that have employed energy restrictions, behavioural change programmes, and demand side management, to encourage more efficient energy behaviour. Despite significant investments in these programmes over the past few decades, more important energy utilization changes are necessary.

\section{Smart Grid Technology}

A smart grid is a solution for improving the infrastructure and electrical energy system in order to create a more intelligent environment. A smart grid is a method for modernizing the electrical power infrastructure and services so that a more smart and dependable power grid can be presented [15]. Traditional grids offer a number of benefits that smart grids do not. Smart grids improve the economic and physical operation of the grid and improve the sustainability and reliability. The market or a smart grid determines an intelligent self-response by integrating sophisticated sensing, measurement and control approaches, and two-way communication into the recent energy system, according to the US Government of Energy's advancing technology grid project. The smart grid concept is depicted in Figure 1 as a block diagram. By establishing a smart power grid, energy security and resource optimization can be improved.

The smart grid system is built to deal with unpredictable events. The smart grid technology is used to maintain power distribution availability based on consumer demand. The major goal of the smart grid is to improve total management that entails gaining direct monitoring of the system and thereby increasing reliability of the system. Despite its low energy efficiency, this approach offers significant advantages in terms of frugality. By utilizing flexible transmission systems, smart grid technologies can assist the system operator in better managing and controlling the energy streams on the grid [16].

The smart grid's distribution network automation enables utility companies to improve the distribution network's strong communication and prevents supply interruptions to end users in the occurrence of unexpected events including an environmental problem that destroys power poles or damages the substation's facilities. Using distribution channel automation also helps to control end-user load. The smart grid's basic mission is to deliver better and more 


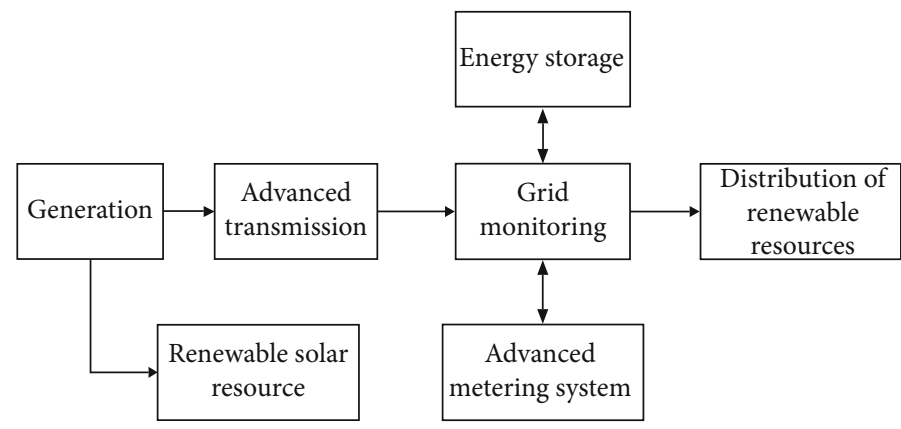

FIGURE 1: Block diagram of smart technology for sustainable resources.

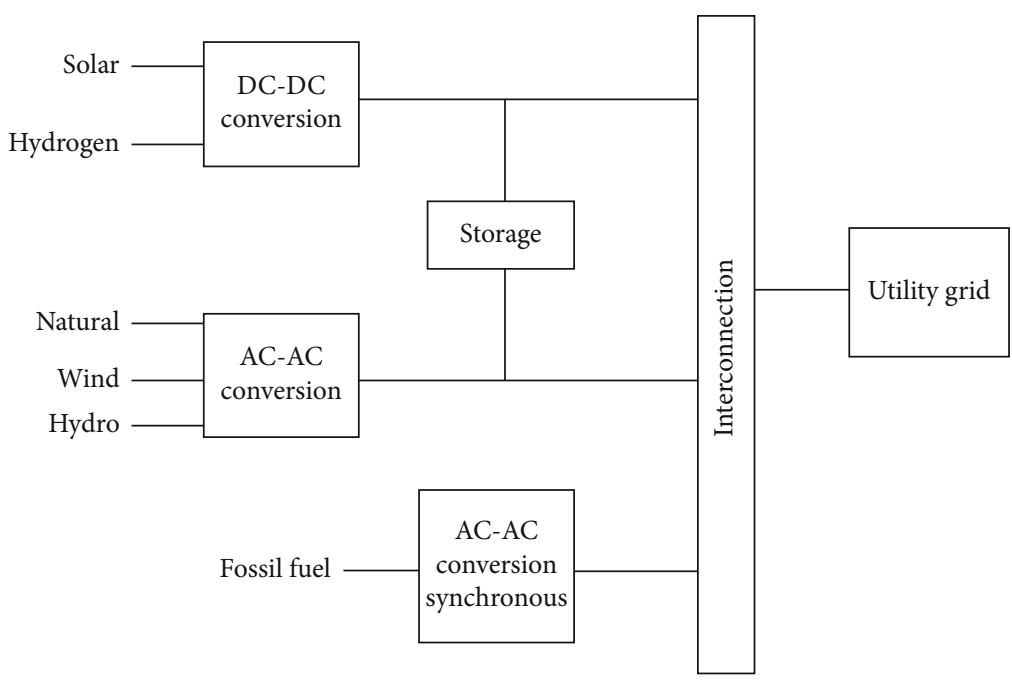

FiguRE 2: Integration of renewable energy resource in grid.

reliable services to end users by integrating current communication systems with diverse grid segments [17].

\subsection{Renewable Energy Source Integration in Smart Grid.} Renewable energy resources are more efficient and convenient, especially in increasing the energy prices and the greenhouse effect. Solar technology is the most perfect system for reducing greenhouse gas emissions and managing energy consumption; it represents a watershed moment in the development of clean and green energy. The most impressive approach is utilized wind generators, solar energy, hydroelectric, and biofuels to generate electricity. The widespread adoption of these types of innovations and technology is contingent on three critical variables connected to future energy supply and grid multiapplication: demand-side capacity planning, distributed energy storage (DES), and DG (DSLM). DG connects multiple energy sources to the electricity grid [18].

Figure 2 reveals the integration of renewable energy resource in grid. In order to establish the smart grid, local power generation from renewable energy is critical. Handling renewable sources of energy necessitate meticulous planning and execution based on technological concepts. A more adaptable, efficient, and smarter grid is required for large-scale distributed renewable energy systems. Energy

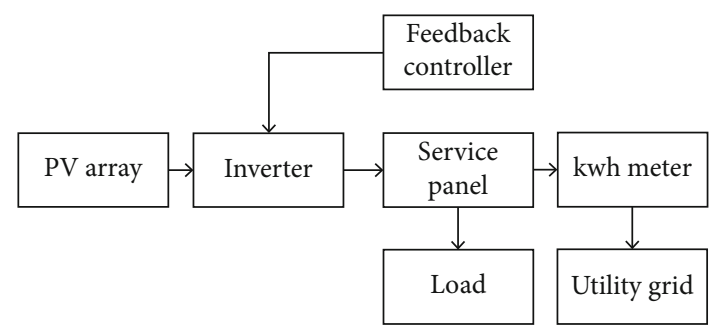

FIgURE 3: Solar energy integration in PV system.

storage is also a key component of the smart grid. The growth of renewable energy sources is aided by the developed system. This system aids in the development of renewable energy sources. The whole renewable energy integration and storage system is depicted in Figure 3. Energy storage will become increasingly important as renewable energy becomes more integrated into the electricity supply system. The usage of a big amount of electrical power storage might be difficult and result in system losses. The usage of DES is a feasible solution since it mitigates the drawback of the smart grid's energy backup requirements [19].

3.2. Solar Energy Utilization. Solar energy is now mostly used to generate electricity using photovoltaic (PV) 


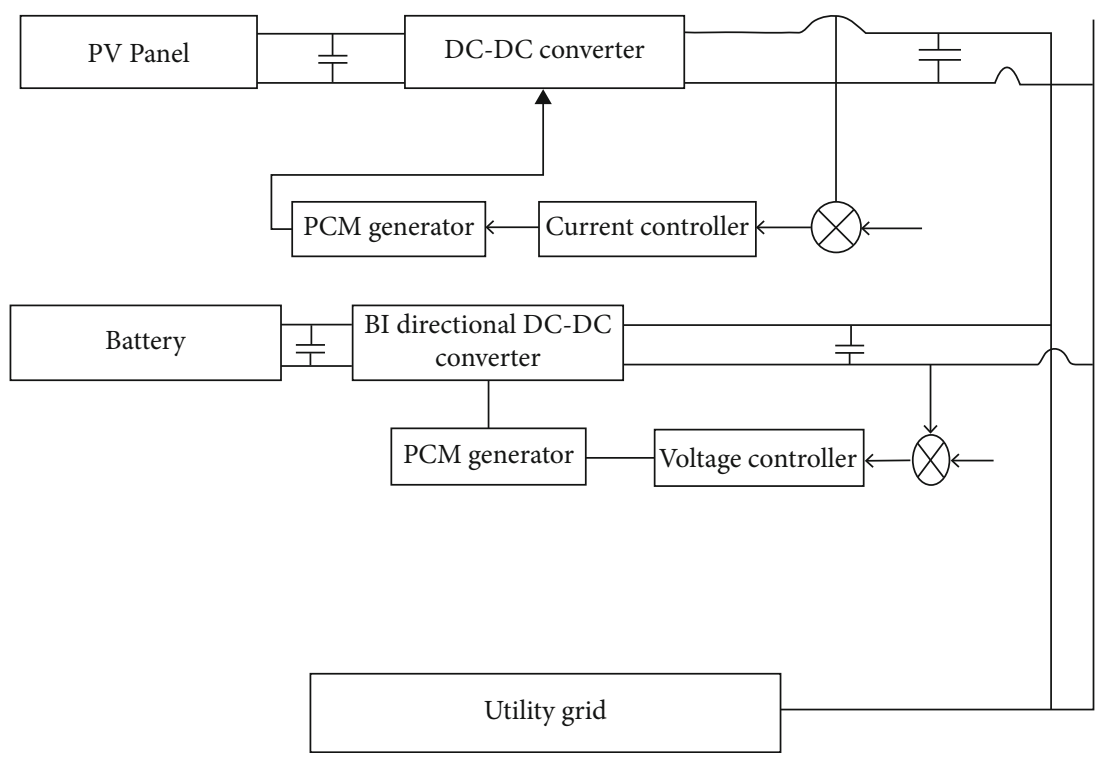

Figure 4: Control scheme connected to renewable resource.

TABLE 1: Energy demand using renewable resource.

\begin{tabular}{lc}
\hline Energy source & Demand (\%) \\
\hline Coal & 22 \\
Gas & 24 \\
Oil & 35 \\
Hydro & 2 \\
Nuclear & 5 \\
Biomass & 10 \\
Other renewable & 1.12 \\
\hline
\end{tabular}

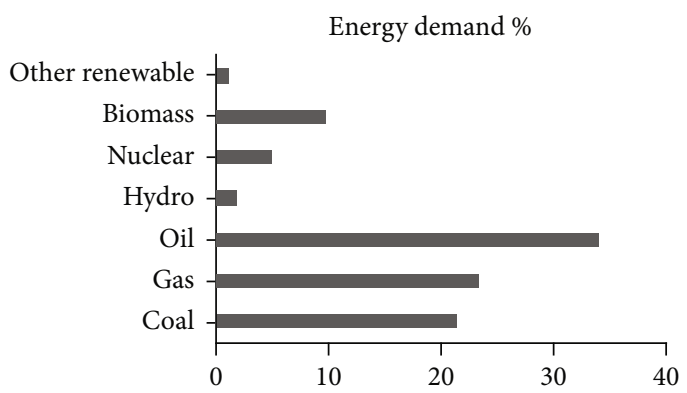

Figure 5: Energy demand for various sources.

TABLE 2: Energy consumption of renewable resources.

\begin{tabular}{lc}
\hline Renewable energy source & Energy consumption \\
\hline Biomass & $13 \%$ \\
Traditional biomass & $61 \%$ \\
Wind & $8 \%$ \\
Hydro & $13 \%$ \\
Biofuel & $5 \%$ \\
\hline
\end{tabular}

technology or heat using various solar-thermal systems. There are other linked photovoltaic-thermal technologies that can produce both thermal and electrical energy, although they are currently less marketable due to the comparatively high total asset cost. The creation of hybrid power systems, depending on the use of solar energy, could be a viable solution for integrating multiple market-available renewable energy sources. The unique control technique allows for the connection of real-time field data with previous usage patterns, resulting in a decrease in energy to imbalance as well as administrative expenses [20].

On a worldwide scale, efficiency improvements of PV systems and their mechanisms lead to increased PV capacity. PV system performance could be improved with correct maximum power point (MMPT) techniques. Solar energy consumption necessitates solar collection and transformation, as well as solar storage. Photovoltaic systems can be used to collect and convert solar energy (PVs). The goal is to decrease the cost per watt of provided solar energy by a factor of ten. Solar energy is diffused associated with fossil energy; thus, material prices must be low to make a photovoltaic process economically viable. If the irradiance striking that area over a time is known, it is quite straightforward to compute the sale price of converted energy that is necessary to pay back at least the original cost of covering a section of the earth with solar energy systems [21].

Because of the variability in local solar irradiance, renewable power will never be a primary energy source for humanity in the lack of expense storage. In theory, batteries might be used to store electricity, but no battery is currently cheap enough to meet the required cost per $W$ requirements for the entire system when amortised over the 30-year lifetime of a solar unit. Electricity might be utilized to power turbines that pump water difficult. This method is comparatively cheap for storage of huge expanses of energy at low adsorption and desorption rates and to charged and discharged every 24 hours to account for the daytime cycle. 
The conversion of sunlight into a "wireless current" is one of the most important phases in photosynthesis. In all cases, $\mathrm{O}_{2}$ must be converted into a usable fuel before being released into our oxygen-rich atmosphere or used as an oxidation reagent for other fuels. The reduced fuel might be hydrogen produced from water reduction or an organic species such as methanol or methane derived from ambient $\mathrm{CO}_{2}$ fixation. The carbon-neutral cycle would subsequently be completed by recombination of the reduced fuel with freed $\mathrm{O}_{2}$. However, considerable advances in basic research are necessary for this technology to attain its full potential.

3.3. Energy Efficiency. Energy sources and supplies are critical for social and economic development. The most important goal is to improve the energy supply system. To improve energy efficiency and technological innovations and capture the dynamic must be to achieve general sustainability objectives for various engineering applications, increased efficiency of efficient estimation available technologies, structures, procedures, or individual components has become a necessity. The continuation of this review paper will present a summary of current breakthroughs in improved efficiency for diverse industrial applications and effective management, as they are critical population issues. Because the topic of efficiency is so broad, the overview offered here is restricted to specific technological difficulties and implementations in general [22].

The effectiveness of different technologies and process elements in energy systems is a prominent research area with substantial implications for sustainability and resource usage. Economic expansion, rising living standards, and societal development in general all come at a cost to the ecosystem, and it is becoming clear that this cost is causing a natural equilibrium to be disrupted. Anthropogenic activities are to a large measure the cause of these problems, and human actions will be required to solve it. Sustainability is a realistic objective that requires a clear vision, meticulous preparation of focused activities, and timely and effective implementation [23].

3.4. Reliability of the Smart Grid. In today's electrical networks, reliability issues are getting more difficult to solve. Grid congestion is worsening, greater transfers are being made over greater distances, variability is rising, and dependability margins are shrinking. The effective information system of islanding coupled to DG has the potential to improve the local energy supply's dependability and quality of service. The user can benefit from an electricity charge based on real-time sensing, which is made feasible by applying advance measuring. The smart grid has features such as self-healing from power outages, allowing end-user contribution in demand managing, cyber-attacks, and physical damage, providing the required power quality in the twenty-first century, flexibility in all generation and storage, and the ability to introduce new products and services [24].

In grid-connected mode, the renewable energy system is illustrated in Figure 4, which must be overseen by certain power converter control schemes to regulate the voltage and power. System synchronisation and management of

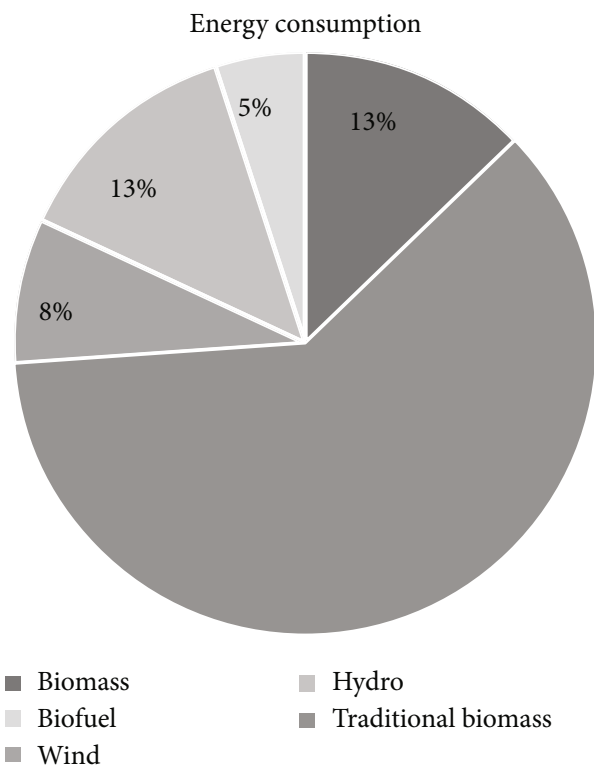

FIgURE 6: Global energy consumption of renewable energy resource.

TABle 3: Annual load distribution for a building for various electrical sources.

\begin{tabular}{|c|c|c|}
\hline & \multicolumn{2}{|c|}{ Energy consumption (\%) } \\
\hline & $\begin{array}{l}\text { Energy } \\
\text { resource }\end{array}$ & $\begin{array}{l}\text { Utilizing renewable energy } \\
\text { resources }\end{array}$ \\
\hline Lighting & 18 & 3 \\
\hline Water heating & 5 & 5 \\
\hline Space heating & 65 & 22 \\
\hline Fan and pump & 12 & 12 \\
\hline $\begin{array}{l}\text { General } \\
\text { purpose }\end{array}$ & 7 & 7 \\
\hline
\end{tabular}

the quality of power put into the grid are the foundations of grid control. The grid functionalities are inserted into Converter Control, which guides the various operating modes, manages the energy stored in the batteries, and provides references for the active and reactive powers.

Smart grid technology controls appliances by delivering power from suppliers to customers via robust twoway digital connections. It is also the integration of communications networks with the electrical power grid in such a way as to create an electricity communications superhighway capable of continuously monitoring its own health, alerting the utility immediately if any problems arise, and automatically taking corrective actions that can allow the grid to fail gracefully and prevent a local failure from spreading out of control. The traditional grid has centralised power generation, unidirectional power flow, and poor market integration at the distribution level. Smart grid technology encompasses both centralised and distributed power generation, with renewable energy sources playing a large role. The system incorporates 


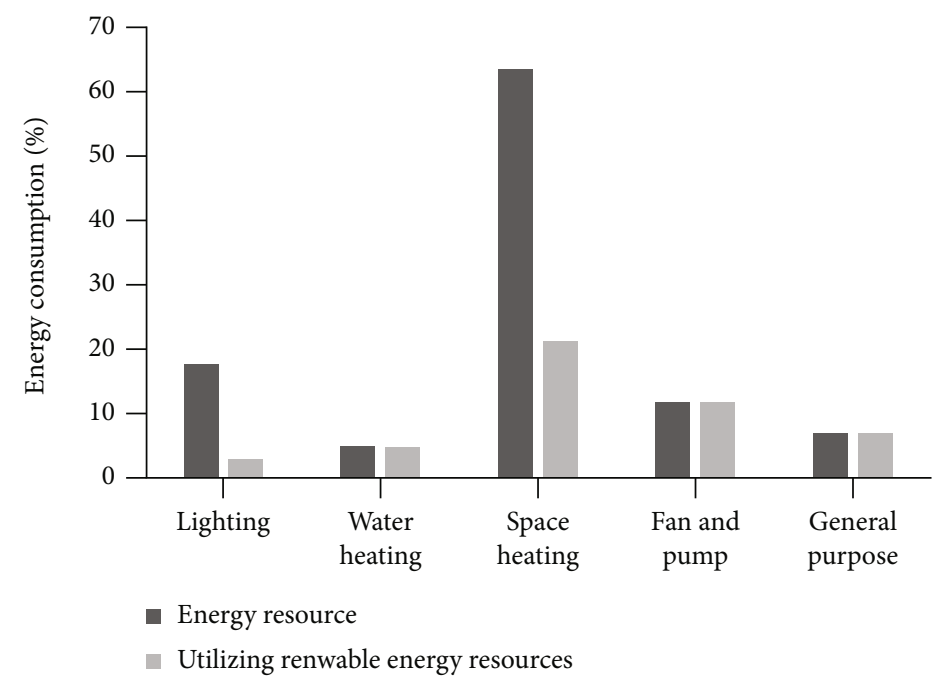

FIGURE 7: Energy efficiency in utilizing renewable resources.

dispersed and active resources such as generation, loads, and storage. It is the electrical network that intelligently connects producers and consumers to effectively supply power that is safe, economical, dependable, efficient, and sustainable. One of the two main objectives for boosting electric power intercommunication for both energy suppliers and end users tends to push smart grid technologies.

Due to ever-increasing energy demand and the objective of reducing greenhouse gas emissions, the world's energy output is changing, necessitating the extensive utilization of renewable energy sources. The renewable energy sources are frequently intermittent and unpredictable, and energy storage is the only answer to this problem. Energy storage is a major aspect in the integration of renewable energy sources, as it helps to increase energy production efficiency while also ensuring a stable and dependable contemporary power grid. It can increase the electric system's flexibility, dependability, and stability by reducing power fluctuations [25].

3.5. Smart Grid Technology and Applications. To construct the smart grid, modern tools and techniques are necessary. To fully comprehend the application of smart grid, it is required to integrate information technology, a robust monitoring system, and a practical strategic strategy. The notion of meeting power demand with a centralised and distributed generation (DG) system using smart grid is a fairly contemporary and trustworthy. Recent technologies and information control the smart grid's system performance and control system, allowing the operator to exercise demand monitoring and conveniently supply dependable and highquality service. Through a two-way communication network based on client answers, the smart grid offers the efficient electrical distribution network. Power companies around the world are unpredictably confronted with significant difficulties. Existing grids are also under pressure to operate securely and reliably. In addition, the social and political benefits of electricity generation and use, as well as their environmental consequences, are significant. Developing
TABle 4: Comparison of smart grid technology with traditional power grid.

\begin{tabular}{lcc}
\hline Criteria & Traditional power grid & Smart grid \\
\hline Metering & Inadequate & Wide \\
Power flow control & Limited & Automated \\
Efficiency & Poor & Excessive \\
Electricity generation & Central & Distributed \\
Restoration & Physical & Self-healing \\
Environmental pollution & High & Low \\
\hline
\end{tabular}

countries need to develop policies based on the need for a more advanced smart grid: a massive oversupply and efficient provision of dependable and high-quality service [26].

\section{Result and Discussion}

Natural gas has a good influence on the environment since it is a cost-effective and efficient source of power. The greatest factor of demand in the energy market will be electric power, indicating an increase in living standards as more individuals and companies have access to safe and dependable electricity. Table 1 reveals the energy demand using renewable resource.

Figure 5 reveals the energy demand for various sources. The world primary energy demand is increasing based on the population rate. The energy consumption shows the increasing of energy demand. The demand of gas is increasing tremendously. The natural renewable resources remain as a fuel for the power generation as it provides more efficiency. The intensities may vary because of differences in economic growth, energy usage, energy rate, location, climate, and culture. Table 2 reveals the energy consumption of renewable resources.

Figure 6 depicts the overall energy contribution of various sectors of energy sources, with a focus on renewable energy sources contribution to total world energy consumption. The renewable energy technology is positive and 
extensive. It is analysed that energy consumption is more in traditional biomass and biofuel provide less energy consumption. The energy consumption profiles for a residential building which have low energy consumption are defined primarily. A number of design factors can impact each primary end use that creates the demand on energy and design variables of building has the possible range of values. Table 3 reveals the annual load distribution for a building for various electrical sources.

Figure 7 shows the annual demand of energy for the building. Various components of the building are used to estimate the total power consumption based on the utilization factor, size, and maintenance factors.

Table 4 reveals the comparison of smart grid technology with the traditional power grid. It is analysed that the smart grid technique is more reliable and flexible and performs better compared to a traditional power grid. The generated power is distributed and provides less pollution to the environment.

\section{Conclusion}

Economic expansion, rising living standards, and societal progress in general all come at a cost to the environment, and it has become clear that this cost is causing a natural equilibrium to be disrupted. Providing energy that is dependable, efficient, and inexpensive is a huge task. Using renewable energy sources to generate power can bring immediate and indirect economic advantages that outweigh the expenses, as well as environmental benefits. Recent advancements in solar energy use have focused on improving existing PV technologies as well as investigating new PV technologies. Improvements in efficiency and the continued expansion of control mechanisms for PVs and energy systems were also explored. A smart strategy enables the integration of data needed to address crucial difficulties in today's societies. The main contest is to limit the properties of global warming while maintaining a healthy economic growth. The smart grid system enhances the efficiency of electricity distribution by addressing the deterioration of energy sources and contemporary information technologies for communication. A smart grid can change the electrical grid of the twentieth century into a network that is more intelligent, adaptable, dependable, self-balancing, and interactive, allowing for economic development, monitoring, energy security, and operational efficiency.

\section{Data Availability}

The data used to support the findings of this study are included within the article.

\section{Conflicts of Interest}

The authors declare that they have no conflicts of interest.

\section{References}

[1] D. Gielen, F. Boshell, D. Saygin, M. D. Bazilian, N. Wagner, and R. Gorini, "The role of renewable energy in the global energy transformation," Energy Strategy Reviews, vol. 24, pp. 38-50, 2019.

[2] M. Bilgili, A. Ozbek, B. Sahin, and A. Kahraman, "An overview of renewable electric power capacity and progress in new technologies in the world," Renewable and Sustainable Energy Reviews, vol. 49, pp. 323-334, 2015.

[3] W. Strielkowski, E. Volkova, L. Pushkareva, and D. Streimikiene, "Innovative policies for energy efficiency and the use of renewables in households," Energies, vol. 12, no. 7, p. 1392, 2019.

[4] Y. Meng, Y. Yang, H. Chung, P.-H. Lee, and C. Shao, "Enhancing sustainability and energy efficiency in smart factories: a review," Sustainability, vol. 10, no. 12, p. 4779, 2018.

[5] I. Vigna, R. Pernetti, W. Pasut, and R. Lollini, "New domain for promoting energy efficiency: energy flexible building cluster," Sustainable Cities and Society, vol. 38, pp. 526-533, 2018.

[6] M. L. Tuballa and M. L. Abundo, "A review of the development of smart grid technologies," Renewable and Sustainable Energy Reviews, vol. 59, pp. 710-725, 2016.

[7] F. M. Bhutta, "Application of smart energy technologies in building sector-future prospects," in 2017 International Conference on Energy Conservation and Efficiency (ICECE), pp. 710, 2017.

[8] M. A. Mac Kinnon, J. Brouwer, and S. Samuelsen, "The role of natural gas and its infrastructure in mitigating greenhouse gas emissions, improving regional air quality, and renewable resource integration," Progress in Energy and Combustion science, vol. 64, pp. 62-92, 2018.

[9] T. R. Matsumoto, S. I. Nabeta, I. E. Chabu, S. Nagao, N. R. Santos, and A. Dominice, "Induction voltage regulator performance in primary distribution networks with a high degree of distributed generation," IEEE Transactions on Power Delivery, vol. 36, no. 3, pp. 1837-1846, 2020.

[10] J. Jackson, "Improving energy efficiency and smart grid program analysis with agent-based end-use forecasting models," Energy Policy, vol. 38, no. 7, pp. 3771-3780, 2010.

[11] A. S. Isa, Y. A. Dodo, H. Ojobo, and I. A. Alkali, "Deployment of smart technologies for improving energy efficiency in office buildings in Nigeria," Journal of Multidisciplinary Engineering Science and Technology, vol. 3, no. 1, 2016.

[12] A. Chel and G. Kaushik, "Renewable energy technologies for sustainable development of energy efficient building," Alexandria Engineering Journal, vol. 57, no. 2, pp. 655-669, 2018.

[13] A. Kalair, N. Abas, M. S. Saleem, A. R. Kalair, and N. Khan, "Role of energy storage systems in energy transition from fossil fuels to renewables," Energy Storage, vol. 3, no. 1, p. e135, 2021.

[14] N. Gaurav, S. Sivasankari, G. Kiran, A. Ninawe, and J. Selvin, "Utilization of bioresources for sustainable biofuels: a review," Renewable and Sustainable Energy Reviews, vol. 73, pp. 205214, 2017.

[15] M. N. Dudin, E. E. Frolova, O. V. Protopopova, O. Mamedov, and S. V. Odintsov, "Study of innovative technologies in the energy industry: nontraditional and renewable energy sources," Entrepreneurship and Sustainability Issues, vol. 6, no. 4, p. 1704, 2019.

[16] M. Saunila, M. Nasiri, J. Ukko, and T. Rantala, "Smart technologies and corporate sustainability: the mediation effect of 
corporate sustainability strategy," Computers in Industry, vol. 108, pp. 178-185, 2019.

[17] G. Dileep, "A survey on smart grid technologies and applications,” Renewable Energy, vol. 146, pp. 2589-2625, 2020.

[18] X. Du, Y. Qi, B. Chen, B. Shan, and X. Liu, "The integration of blockchain technology and smart grid: framework and application," Mathematical Problems in Engineering, vol. 2021, Article ID 9956385, 12 pages, 2021.

[19] G. L. P. Raj and V. Kirubakaran, "Energy efficiency enhancement and climate change mitigations of SMEs through gridinteractive solar photovoltaic system," International Journal of Photoenergy, 2021.

[20] C. Ocampo-Martinez, "Energy efficiency in discretemanufacturing systems: insights, trends, and control strategies," Journal of Manufacturing Systems, vol. 52, pp. 131145, 2019.

[21] M. J. Khan and Pushparaj, "A novel artificial intelligence based hybrid maximum power point tracking technique for solar photovoltaic system," Journal of Electrical Engineering \& Technology, vol. 16, pp. 1879-1889, 2021.

[22] S. Nižetić, N. Djilali, A. Papadopoulos, and J. J. Rodrigues, "Smart technologies for promotion of energy efficiency, utilization of sustainable resources and waste management," Journal of Cleaner Production, vol. 231, pp. 565-591, 2019.

[23] M. A. Ahad, S. Paiva, G. Tripathi, and N. Feroz, "Enabling technologies and sustainable smart cities," Sustainable Cities and Society, vol. 61, p. 102301, 2020.

[24] R. Sharma and H. Kamth, "Reliability analysis for smart grid challenges in India," in 2018 International Conference on Circuits and Systems in Digital Enterprise Technology (ICCSDET), pp. 1-3, 2018.

[25] E. Iacovidou, P. Purnell, and M. K. Lim, "The use of smart technologies in enabling construction components reuse: a viable method or a problem creating solution?," Journal of Environmental Management, vol. 216, pp. 214-223, 2018.

[26] B. K. Bose, "Power semiconductor devices for smart grid and renewable energy systems," Power Electronics in Renewable Energy Systems and Smart Grid: Technology and Applications, IEEE, pp. 85-152, 2019. 